\title{
MASTER-SLAVE SYSTEM WITH TELEOPERATION FOR REHABILITATION
}

\author{
Minh Duc Duong* Chisato Teraoka* \\ Takashi Imamura* Takanori Miyoshi* \\ Kazuhiko Terashima* \\ * Toyohashi University of Technology \\ Hibarigaoka 1-1, Tempaku-cho, Toyohashi, 441-8580 Japan
}

\begin{abstract}
In this paper, a master-slave robot system with haptic feedback, human sensing and teleoperation for rehabilitation of upper limb function is presented. The physician could adjust on-line the exercise based on the EMG signal of the patient and force feedback. Moreover, in the present teleoperating system, it is shown that the stability is guaranteed due to the passivity of the master arm's mechanism, in spite of the communication time delay. Copyright@2005 IFAC
\end{abstract}

Keywords: Master-slave systems, Teleoperation, Time delay, Lyapunov stability, Pulse width modulation

\section{INTRODUCTION}

Japan is gradually becoming a more elderly society, and the consequently the number of people who need some kind of medical care is increasing. At the same time, people of all ages continue to require assistance recovering from accidents, congenital problems, or chronic illness. However, the number of physicians and nurses is not increasing in step with the increasing number of patients. Therefore, it is necessary to develop automated systems for patient care. One alternative that is attracting a lot of interest is the use of welfare or life-supporting robots.

In recent years, many studies have focused on the rehabilitation of upper limb function. Among the proposed systems is a haptic interface arm (Loureiro et al., 2003), in which patients move the arm and interact with a virtual environment. In this system, a spring-damper model is used to help the patient follow the exercises. The patient's position, velocity and force data are all collected and analyzed. Another system that uses speed, time or the EMG (Electromyography) threshold to initiate robot assistance for maximizing the patient's recovery has been proposed (Krebs et al., 2003). During the patient's performance, speed of movement, time or the EMG signal of patients are measured and evaluated to find a threshold value. Patients perform the exercise without any assistance until they reach their thresholds; then the assistant robot is engaged. In both systems, the exercises are programmed into the computer beforehand and the patients practice the exercise as if they were playing a simple computer game. Other rehabilitation systems have also been developed ((Burgar et al., 2000); (Koyanagi et al., 2003)). Those systems supply the exercises for patients that assist them in daily life activities. The trajectories that the patient's arm must follow are also programmed beforehand. Depending on the patient's condition and the exercise, the system will assist or guide the patient using the difference between the real position and desired position. The disadvantage of all the above systems is that they have static characteristics. This means that they are set before starting their work, 
and the setting can only be changed when the task finished. As a result, either the physician must do a lot of work to adjust these machines for each patient, or the exercises must be simple enough to accommodate all patients.

Master-slave systems, in which the physician is supported to work more comfortably, are good instruments for rehabilitation. Moreover, by teleoperation, the physician and patient can be in different places. Force feedback technology provides good information for the master-slave system. However, it also makes the system become unstable, especially in case of teleoperation with communication time delay. By using passivity and scattering theory, it was shown that the non-passivity of the communication environment causes the system's instability; subsequently, a control law to solve the instability problem was proposed (Anderson and Spong, 1989). The same result was also achieved using wave variables (Niemeyer and Slotine, 1991). However, those results are only useful in the case of constant time delay. When it became clear that variable time delay threatened the system stability, a solution for stability in a variable time delay environment was proposed (Lozano et al., 2002). Further improvements were made, such as improving position tracking (Chopra et al., 2004) and discrete-time implementation of the control law (Berestesky et al., 2004). The limit in this solution is that the derivative of time delay $\mathrm{T}$ over time must be less than one, $d T / d t<1$. Using a four-channel data transmission structure, the stability and transparency of a master-slave system could be achieved (Zhu and Salcudean, 1995), assuming that the operator and the environment's model are known. However, implementation of force feedback with varying time delay and an unknown environment's model is still a problem.

In this paper, two degree of freedom (2-DOF) master-slave robot arm system is proposed with haptic feedback, human sensing and teleoperation for rehabilitation. The slave robot arm is designed to match a human arm, and the master robot arm is built upon a similar structure but on a smaller scale than the slave arm. This makes it easier for the physician to operate the exercise at lower energy, compared to performing the exercise by holding the patient's arm directly. The patient's conditions can be transmitted to the physician using force feedback and EMG signals. Using a haptic feedback signal and EMG signal, the physician can adjust the exercise for the patient in real-time. In addition, tele-rehabilitation (rehabilitation by teleoperation) is implemented in the system where the master site and the slave site are connected with each other through communication environment with time delay. To avoid the instability problem in our system, instead of using actuators in both master and slave sites such as other master-slave systems, we use electromagnetic brakes at the master site. Brakes can communicate a patient's resistance to the master arm's movement, and also stabilize the system in a communication environment with time delay.

\section{SYSTEM STRUCTURE AND OPERATION}

The master-slave system built in this research includes a 2-DOF master robot arm and 2-DOF slave robot arm. They have similar structures, but the master arm's size is smaller as shown in Table I. The master arm uses encoders to give its position to the slave arm. Instead of actuators, the master arm has brakes for the implementation of feedback force. Force feedback can be realized by attaching the force strain gauge to the handle part of the slave arm. The slave arm has two DC motors with encoders and reduction gear with a 1:50 ratio. Two ball reducers with a 1:20 ratio are used in two joints in slave arm. A controller is designed for the slave arm in order that the slave arm can track the master arm's movement. Master and slave arms connect with each other through a communication environment specified by more or less time delay. A schematic diagram and specification of the system are shown in figure 1 and Table 1 respectively. In addition, in this system, the EMG signal of the patient's arm is also measured and then monitored. The physician can observe the EMG signal in the computer display at the master site.

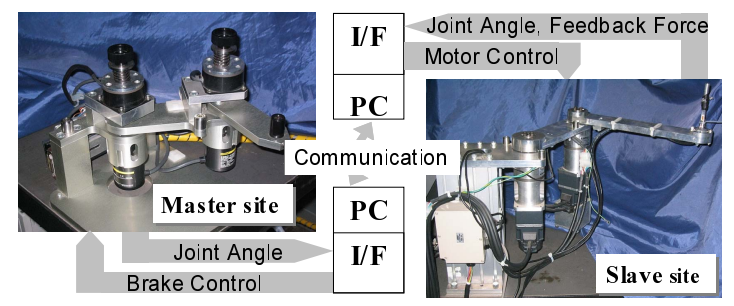

Fig. 1. Schematic diagram of master-slave telerehabilitation system

Table 1. Specifications of the master arm and the slave arm

\begin{tabular}{ccc} 
Elements & Master arm & Slave arm \\
\hline Size(W:D:H) $[\mathrm{mm}]$ & $195: 195: 210$ & $165: 215: 300$ \\
Arm1 length[mm] & 120 & 200 \\
Arm2 length[mm] & 115 & 300 \\
Weight[kg] & 2.0 & 30.0 \\
Actuator & Brake & DC Motor \\
Encoder[ppr] & 2000 & 500 \\
Material & Aluminum alloy & Aluminum alloy \\
\hline
\end{tabular}

The system operates as follows: The operator (physician) moves the master arm, and the joint angles of master arm are measured. Then, the position information is sent to the slave site. At 
the slave site, the slave arm is controlled to track the master arm's movement. The patient holds the handle attached to the tip of the slave arm, and therefore, follows the master arm's movement. If the patient feels pain from the exercise, he or she will resist the slave motion. The force sensor attached to the handle measures the force when the patient pulls or pushes the handle. Then, force information is sent back to the master site. The master site receives the force signal and the brake at each joint of master arm is applied. These brakes then actively resist the master arm movement made by the physician. The EMG signal of patient's arm is also measured and sent to the master site so that the physician can better grasp the patient's condition. By feeling the resistance and observing the EMG signal, the physician will adjust exercise to be appropriate for the patient in real-time.

\section{DYNAMICS OF THE SYSTEM}

\subsection{Master site}

The equation for the master arm model can be represented by

$$
J_{m i} \ddot{\theta}_{m i}=T_{m i}
$$

, where $J_{m i}$ is the inertia, $\theta_{m i}$ is the angle, and $T_{m i}$ is the total torque (including the operator torque $T_{h i}$ and the brake torque $\left.T_{b i}\right)$ of $i^{\text {th }}$ joint $(\mathrm{i}=1,2)$.

$$
T_{m i}=T_{h i}-T_{b i}
$$

In the master arm, two brakes are prepared to adjust impedance of the master arm's movement, and the actuators to move the master arm is not used. Brakes only resist the movement. Therefore, the brake torque $T_{b i}$ depends on not only the feedback torque value from the slave site, but also the sign of velocity and operator torque. $T_{b i}$ can be expressed as

$$
\text { - } \dot{\theta}_{m i} \neq 0
$$

$$
T_{b i}=\operatorname{sign}\left(\dot{\theta}_{m i}\right)\left|T_{f i}\right|
$$

- $\dot{\theta}_{m i}=0$

$$
T_{b i}= \begin{cases}\operatorname{sign}\left(T_{h i}\right)\left|T_{f i}\right| & \text { if }\left|T_{h i}\right|>\left|T_{f i}\right| \\ T_{h i} & \text { if }\left|T_{h i}\right| \leq\left|T_{f i}\right|\end{cases}
$$

, where $T_{f i}$ is the feedback torque from the slave site at the $i^{t h}$ joint of the master arm $(\mathrm{i}=1,2)$.

\subsection{Slave site}

Suppose that the model of each joint of the slave arm is a first-order system model as shown in the following:

$$
\frac{\dot{\theta}_{s i}(s)}{V_{s i}(s)}=\frac{K_{s i}}{T_{s i} s+1}
$$

, where $\theta_{s i}$ is the angle, and $V_{s i}$ is the input voltage of the motor at the $i^{\text {th }}$ joint. $K_{s i}$ and $T_{s i}$ are the coefficients of the transfer functions $(i=1,2)$.

In order to make the slave arm track the master arm's movement, a PD (Proportional-Derivative) controller is used. The PD controller can be expressed as follows:

$$
V_{s i}=K_{P i}\left(\theta_{s d i}-\theta_{s i}\right)+K_{D i} \frac{d}{d t}\left(\theta_{s d i}-\theta_{s i}\right)
$$

, where $V_{s i}$ is the output of the $\mathrm{PD}$ controller and also the input of the slave motors, $\theta_{s d i}$ is the desired angle, $\theta_{s i}$ is the slave joint angle, $K_{P i}$ is the proportional gain, and $K_{D i}$ is the derivative gain at the $i^{t h}$ joint of the slave arm $(\mathrm{i}=1,2)$.

\subsection{Brake characteristics}

In our system, electromagnetic brakes are used at the master site to implement the feedback force from the slave site so that the physician can feel the patient's resistance. The brake torque is controlled to be proportional to the resist force and therefore, proportional to the magnitude of the strain gauge signal. The brake torque can be controlled by input voltage. However, as shown in figure 2 , the relation between input voltage and brake torque in the case of increasing (circle) and decreasing (triangle) voltage, are not similar and nonlinear. In those circumstances, the brakes cannot be controlled directly by the input voltage proportional to the strain gauge signal.

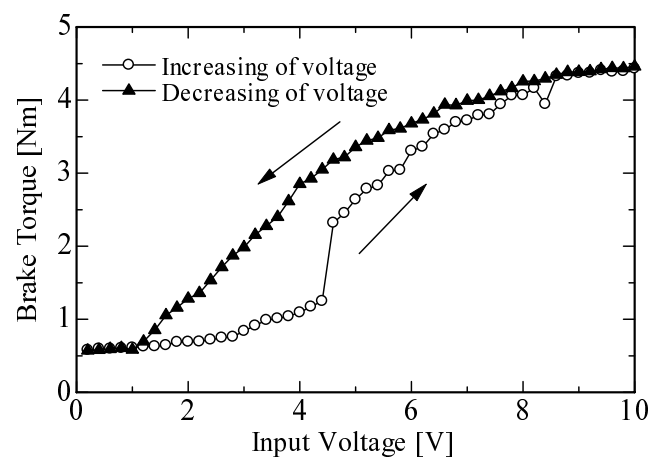

Fig. 2. Relation between input voltage and brake torque

In order to improve the brake characteristics, the PWM (Pulse Width Modulation) method was used. Instead of generating the voltage with a magnitude proportional to the desired value, a pulse with constant magnitude and pulse width proportional to the desired value is generated in each PWM cycle. As shown in figure 3, the relation between duty cycle and brake torque is 


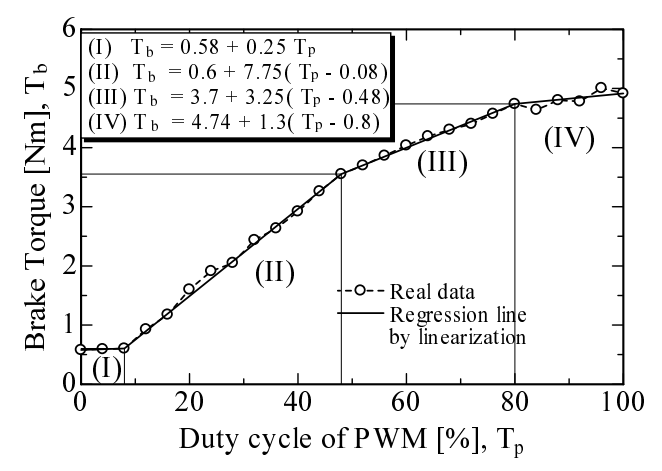

Fig. 3. Relation between PWM duty cycle and brake torque

the same in both an increasing (dashed line) and decreasing (circle) duty cycle. The characteristic line is still nonlinear, but it can be divided into four linear parts (solid line in figure 3). Thus, linearity between the resist force at the slave arm and brake torque at the master arm can be achieved.

\section{MEASUREMENT OF HUMAN MUSCLE POWER BY EMG SIGNAL}

In our rehabilitation system, sensing functions that can measure non-verbal information about the patient are needed. This information is provided so that the physician may observe the patient's conditions. It is also necessary to achieve safety in tele-rehabilitation. In order to apply sensing functions to the rehabilitation system, data such as the patient's EMG, heart rate, blood pressure, and so on must be collected by the computer in real-time and must be processed for easy observation and evaluation. In this paper, the EMG signals were taken up as biological information from the viewpoint of rehabilitation exercises for the upper limbs.

Sample data of EMG signals and the angle of the elbow joint are shown in figure 4 at the case of exercise for the bending and stretching of the elbow joint. The setup of the measurement of the EMG signal is also shown in figure 5. In this experiment, four surface electrodes were placed on the (1)Biceps brachii, (2)Triceps brachii, (3)Flexor carpi radialis and (4)Extensor carpi radialis (longus and brevis), and the elbow angle was measured by the goniometer. The sample time in this experiment was $1[\mathrm{~ms}]$.

Figure 4 shows that the amplitude of the EMG signal at the Biceps brachii is enlarged by the bending movement of the patient's elbow. On the other hand, the amplitude of EMG signal at the Triceps brachii is enlarged by the extension movement of the patient's elbow. These relationships between the amplitude of the EMG and movement of elbow show the good explanations for the structure of the muscles in sports physiology.

Theoretically, the EMG signal amplitude and frequency will change depending on the conditions of the muscle, for example, whether the muscle is fatigued. Therefore, the EMG signal should be useful for the observation of patient in rehabilitation with the support of several signal processing methods in order to specify the desired characteristics of the muscles.

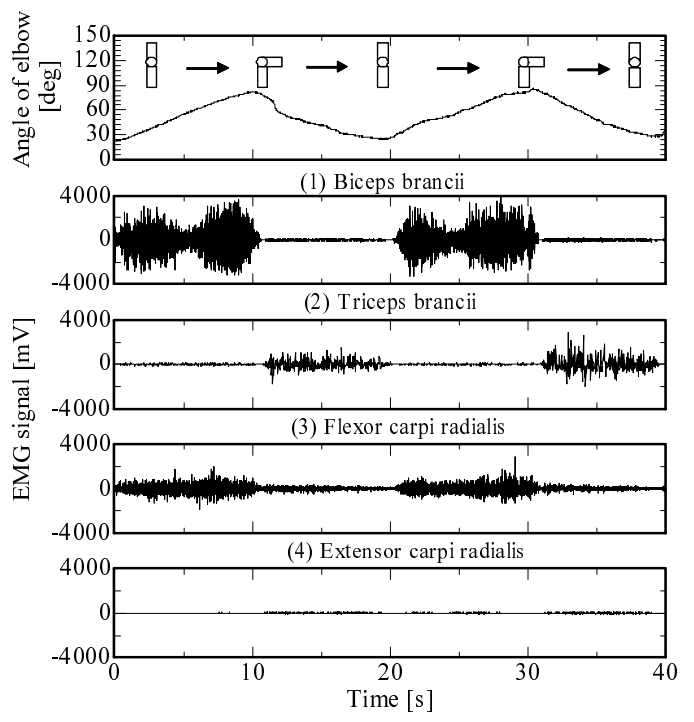

Fig. 4. EMG signal of human muscles

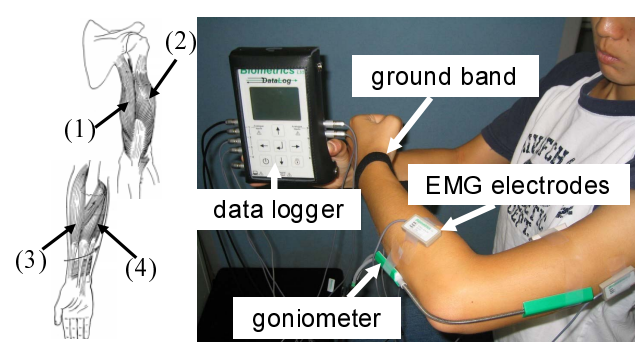

Fig. 5. Setup of measurement system of EMG signal

\section{TELEOPERATION OF THE MASTER-SLAVE SYSTEM}

Teleoperation can overcome the distance problem because the master site and slave site can be remote from one another. In teleoperation, feedback information is very important. It can improve the effectiveness of the tasks, especially in rehabilitation machines; feedback information can allow the physician to adjust the exercise depending on the patient's condition. However, force feedback may also make the system become unstable. 


\subsection{Stabilization of the teleoperation system}

Force feedback from the slave site to the master site makes the system become a closed-loop system. Therefore, the time delay by teleoperation can cause the instability of the closed-loop system. As mentioned in the introduction, many researchers have tried to establish the stability of the teleoperation system with force feedback in various conditions.

In our system, instead of installing actuators in the master site as other researchers have done to achieve bilateral control, we use brake mechanisms at the master site such that the physician can feel the patient's condition and the patient's safety can be maintained by putting a brake on the master's movement, exerting strong impedance to the physician. This system alerts the physician to the potential danger at his or her hand. The system is still a closed-loop system, as shown in figure 6 ; therefore, the instability caused by time delay may happen. However, our system can solve this problem. The explanation is as follows:

Considering the closed-loop system with human torque $T_{h i}$ as the input and the master arm's velocity $\dot{\theta}_{m i}$ as the output, the slave site, communication environment, and brake are in the feedback path.

The Storage function $\mathrm{S}$ of the system is

$$
S=\frac{1}{2} J_{m i} \dot{\theta}_{m i}^{2}
$$

$\mathrm{S}$ is positive defined function. Then, $\dot{S}$ is calculated using (1) and (2) as follows:

$$
\dot{S}=J_{m i} \ddot{\theta}_{m i} \dot{\theta}_{m i}=T_{h i} \dot{\theta}_{m i}-T_{b i} \dot{\theta}_{m i}
$$

From (3) and (4),we have

$$
T_{b i} \dot{\theta}_{m i}= \begin{cases}\operatorname{sign}\left(\dot{\theta}_{m i}\right) \dot{\theta}_{m i}\left|T_{f i}\right| \geq 0 & \text { if } \dot{\theta}_{m i} \neq 0 \\ 0 & \text { if } \dot{\theta}_{m i}=0\end{cases}
$$

$T_{b i} \dot{\theta}_{m i} \geq 0$ with any value of $T_{f i}$. Therefore

$$
\dot{S}=T_{h i} \dot{\theta}_{m i}-T_{b i} \dot{\theta}_{m i} \leq T_{h i} \dot{\theta}_{m i}
$$

This means that the system is passive for any value of $T_{f i}$. The brakes make the system become passive in spite of the time delay.

In other words, by setting $T_{h i}=0$, then $\dot{S} \leq$ $T_{h i} \dot{\theta}_{m i}=0 . \mathrm{S}$ is a Lyapunov function for the system, therefore the system is stable.

\subsection{Experimental setup and results}

To examine the stability of our system, experiments were implemented. A block diagram of the system is shown in figure 6 with the behavior of communication block being

$$
\theta_{s d i}=\theta_{m i}(t-T), V_{b i}=V_{g i}(t-T)
$$

where $\mathrm{T}$ is the communication time delay, $V_{g i}$ is the strain gauge signal and $V_{b i}$ is the control signal of the $i^{t h}$ brake $(\mathrm{i}=1,2)$.

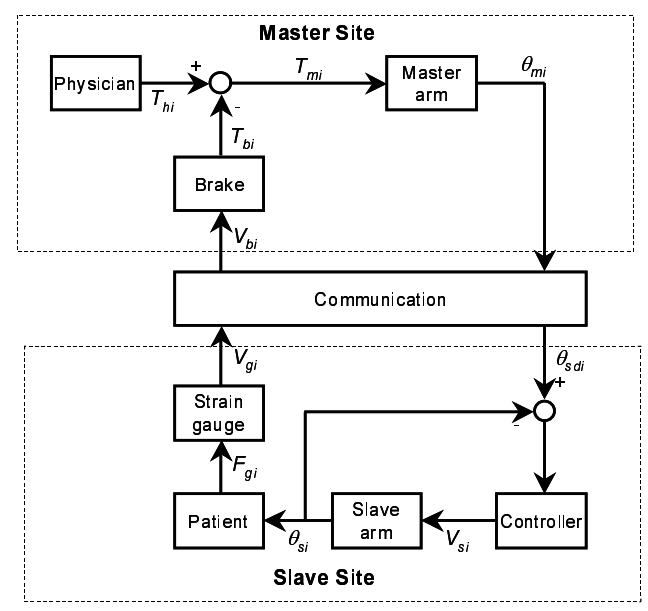

Fig. 6. Block diagram of the master-slave telerehabilitation robot system

The experimental setup was as follows:

- For simplicity's sake, the experiment was only for one joint of the master-slave system. In this system, because of the structure similarity, each joint in the slave arm was controlled independently to track the motion of the respective joint in the master arm. Therefore, we implemented teleoperation with one joint, assuming that and the result could be applied to the whole system.

- In the first experiment, the response of patient was considered to be like a spring system where the measured force at the strain gauge is proportional to joint's angle. The response differed from person to person and it was difficult to model. In this experiment, we considered the following situation for the patient's rehabilitation. If the patient's arm is still moved when the patient start feeling pain, he or she will feel more pain, and will put more force to the handle at the slave arm. We suppose that the response of patients acts like a spring system.

- In the second experiment, a person held the handle of slave arm and suddenly resisted the slave arm's motion. We considered the situation in which the patient feels pain and he or she wants to reduce or stop the movement.

- The brake torque is also proportional to the measured force at the strain gauge.

- The communication time delay between the master site and the slave site is constant.

The result of the first experiment is shown in figure 7 . The system was stable and the slave position tracked the master position regardless of the large delay of $\mathrm{T}=1[\mathrm{~s}]$. When the resistance force at the slave site expressed by the strain gauge signal increased, the physician had to generate more 
force to move the master arm. The physician could feel the patient's resistance through the brake.

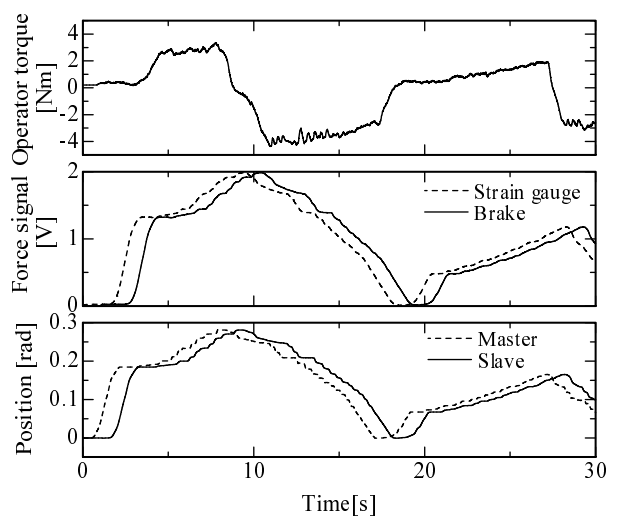

Fig. 7. First experimental results of master-slave tele-rehabilitation with time delay of $1[\mathrm{~s}]$

The second experiment's result is shown in figure 8. Stability and good tracking were also achieved here. In addition, when the patient pushed or pulled the handle in the slave arm by a strong enough force, it was difficult for the physician to move the master arm, even with a strong force. The brake obstructed the physician's movement in order to ensure safety for patient.

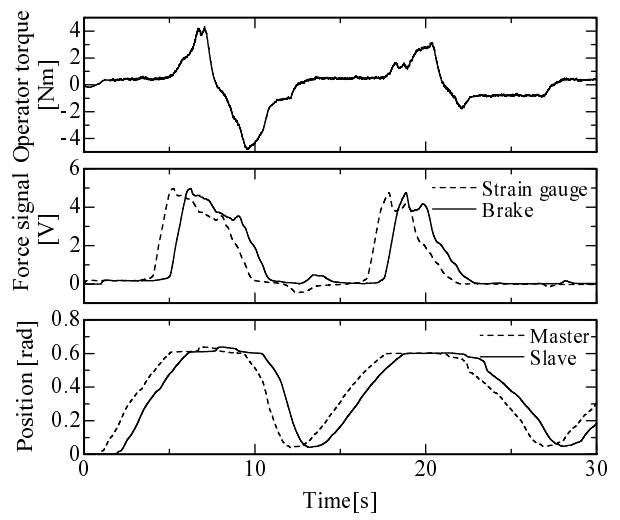

Fig. 8. Second experimental results of masterslave tele-rehabilitation with time delay of $1[\mathrm{~s}]$

\section{CONCLUSION}

In this paper, a master-slave robot system with haptic feedback and teleoperation for rehabilitation was built. Using this system, a physician can observe the EMG signal and feel the resistance of the patient in order to evaluate the patient's state and adjust on-line the exercise. Moreover, the stability of the system with teleoperation and force feedback was guaranteed by using brakes, instead of actuators, in the master arm.
This research is fundamental in establishing a tete-rehabilitation system using human sensing information such as EMG, pain level, heart rate, blood pressure, autonomous nerve functions, etc. This challenging work is just beginning, and will require extensive further research.

\section{ACKNOWLEDGMENT}

We greatly appreciate the support of City Area Project "Smart Sensing" by the Japanese Ministry of Education, Culture, Sports, Science and Technology.

\section{REFERENCES}

Anderson, R. J. and M. W. Spong (1989). Bilateral control of teleoperators with time delay. IEEE Trans. on Automatic Control.

Berestesky, P., N. Chopra and M. W. Spong (2004). Discrete time passivity in bilateral teleoperation over the internet. IEEE Int. Conf. on Robotics and Automation.

Burgar, C. G., P. S. Lum, P. C. Shor and H. F. Machiel Van der Loos (2000). Development of robots for rehabilitation therapy: The palo alto va/stanford experience. Journal of Rehabilitation Research and Development.

Chopra, N., M.W. Spong, R. Ortega and N.E. Barabanov (2004). On position tracking in bilateral teleoperation. American Control Conference.

Koyanagi, K., J. Furusho, U. Ryu and A. Inoue (2003). Development of rehabilitation system for the upper limbs in a nedo project. Proc. of the 2003 IEEE Int. Conf. on Robotics and Automation.

Krebs, H. I., J. J. Palazzolo, L. Dipietro, M. Ferraro, J. Krol, K. Rannekleiv, B. T. Volpe and N. Hogan (2003). Rehabilitation robotics: Performance-based progressive robot-assist therapy. Autonomous Robots 15 pp. 7-20.

Loureiro, R., F. Amirabdollahian, M. Topping, B. Driessen and W. Harwin (2003). Upper limb robot mediated stroke therapy-gentle/s approach. Autonomous Robots 15 pp. 35-51.

Lozano, R., N. Chopra and M. W. Spong (2002). Passivation of force reflecting bilateral teleoperators with time varying delay. Mechatronics'02.

Niemeyer, G. and Jean-Jacques E. Slotine (1991). Stable adaptive teleoperation. IEEE Jounal of Oceanic Engineering.

Zhu, M. and S. Salcudean (1995). Achieving transparency for teleoperator systems under position and rate control. 1995 IEEE/RSJ Int. Conf. on Intelligent Robots and Systems (IROS'95). 